\title{
SPINNING OPTICAL SPATIOTEMPORAL SOLITONS IN QUADRATIC MEDIA
}

\author{
D. Mihalache \\ Department of Theoretical Physics, Institute of Atomic Physics \\ P.O. Box MG-6, Bucharest, Romania
}

\begin{abstract}
The unique features of the families of bright spinning spatiotemporal solitons (doughnuts or vortex light bullets) in dispersive quadratic media, including their stability, are presented. Both analytical results, obtained by means of a simple variational approximation, and numerical simulations are presented and compared. It was found that though the variational approximation is not very accurate, it correctly describes the qualitative features of the spinning spatiotemporal solitons. The spinning light bullets are subject to a strong azimuthal instability, which leads to the break-up of the spinning soliton into a set of fragments, each being a stable nonspinning spatiotemporal soliton.
\end{abstract}

PACS numbers: 42.65.Tg, 42.65.Sf

\section{Introduction}

Solitons in optical media with quadratic nonlinearities exhibit unique dynamical behaviors and have a potential for applications to photonic devices [1-20]. One of the fundamentally important properties is the fact that, unlike the Kerr nonlinearity [21], the quadratic nonlinearity does not lead to wave collapse in any physical dimension [3], and thus it opens a way to generate stable spatiotemporal solitons (STS), or "light bullets" (LB) [21], i.e., fully localized spatiotemporal objects that result from the simultaneous balance of diffraction and dispersion by nonlinear phase-modulation. STS in various types of nonlinear optical environments have attracted a great deal of interest [22-42]. However, collapse does not take place, making stable LB possible, in media with saturable [25, 26, 31], quadratic [35, 39], and cubic-quintic [43,44] nonlinearities, in off-resonance two-level systems [41] as well as in self-induced-transparency media [40]. Recently in Ref. [41] the propagation of 2D femtosecond optical pulses in an off-resonance two-level medium was addressed. Within the quasiadiabating following approach, the evolution of the pulse is governed by a generalized Kadomtsev-Petviashvili equation with coupling 
between the spatial and temporal profile. It was shown that due to the interaction between the transient, retarded diffraction and the electromagnetic absorption, stable, localized (2+1)-dimensional optical pulses can be formed. We mention also that the possibility of forming spatiotemporal solitons in inhomogeneous, dispersive nonlinear media using a graded-index Kerr medium as an example was investigated via a variational approach [42].

On the one hand, STS is a challenging object for fundamental research, as examples of stable localized objects in two-dimensional (2D) and, especially, in three-dimensional (3D) nonlinear media are rare in physics. On the other hand, STS hold promise for potential applications in future ultrafast all-optical logic devices, where each STS may represent an elementary bit of information, provided that stable STS can be formed from pulses at reasonable energy levels in available optical materials.

The formation of 2D STS in quadratically nonlinear media has been recently observed [45]. In these experiments, tilted-pulse techniques were used to control the effective group-velocity-dispersion (GVD) and group-velocity-mismatch (GVM) experienced by the propagating signals [46]. In particular, the effective GVD was made anomalous and properly enhanced, while the GVM was reduced. An important peculiarity of the results reported in [45] is that 2D STS can be successfully generated despite a nonvanishing value of group-velocity-mismatch between fundamental-harmonic (FH) and second-harmonic (SH) waves. Very recently, noncollinear generation of optical 2D STS, based on type-I interaction (the one which involves a single FH wave) in quadratically nonlinear media has been demonstrated in a barium metaborate $\left(\mathrm{Ba}_{2} \mathrm{BO}_{4}\right.$, alias $\left.\mathrm{BBO}\right)$ crystal [47]. The resulting Y-like geometry of the optical STS generation can be used to implement optical logical AND gates with ultrafast high-contrast operation. However, 3D STS in quadratically nonlinear media have not been observed experimentally thus far.

An important feature that must be taken into regard in any physically realistic model of second-harmonic generation ( $\mathrm{SHG}$ ) media is the fact that the FH and SH waves have different dispersions: while the dispersion at both frequencies must be anomalous to support fully stationary STS [35], their absolute values are, generally, different. This implies that equations describing the structure of the STS include a spatiotemporal anisotropy which has no analog in the case of spatial solitons [35].

Static (nonwalking) STS [35-39] are represented by real solutions to the corresponding coupled nonlinear wave equations. In this case, STS actually move at a velocity exactly equal to the group velocity of the carrier waves. In the SHG model, it is usually assumed that FH and SH group velocities coincide. Then, the only free parameter of the STS solutions is their propagation constant (nonlinear wave number shift). An important generalization to the case of "walking" STS, represented by complex (chirped) stationary solutions, which move at a finite velocity relative to the carrier-wave group velocity, was recently carried out for a simpler $2 \mathrm{D}$ case [48]. Such a generalization was necessary, first of all, because, in reality, the $\mathrm{FH}$ and $\mathrm{SH}$ group velocities are not exactly equal. Moreover, in a real experimental situation, the mismatch between the two group velocities may be significant [45], which does not prevent the formation of quadratic solitons 
when the phase-mismatch between the waves is correspondingly large [49], but makes a detailed theoretical study of the "walking" effects necessary. Families of 1D "walking solitons" were studied in detail as solitary-wave solutions of the SHG models in the presence of spatial or temporal walk-off [16-18]. It has been found that the walking solitons have features essentially different from those of the static ones, e.g., a different energy distribution between their FH and SH components, and different soliton contents [50] produced by arbitrary (non-soliton) input pulses.

Next we discuss in some detail recent numerical work on one-parameter families of 3D spinning (vortex) LB in a model of a type-I SHG medium [51]. The model assumes different coefficients of the GVD at the FH and the SH [35] but neglects the Poynting-vector walkoff and temporal group-velocity mismatch. Spinning LB in models of this type were very recently considered in a brief form in Ref. [52], using a variational approximation and very limited numerical computations. Here we compare, for a selected set of parameters, the exact numerical results with those obtained by using a simple variational method. However, a crucially important issue is the (in)stability of the spinning LB against azimuthal perturbations which will be considered too.

\section{Spinning light bullets}

The scaled equations describing type-I SHG processes (i.e., involving a single $\mathrm{FH}$ polarization) in the $(3+1) \mathrm{D}$ geometry in the presence of dispersion and diffraction are well known [35]

$$
\begin{aligned}
& \mathrm{i} \frac{\partial u}{\partial Z}+\frac{1}{2}\left(\frac{\partial^{2} u}{\partial X^{2}}+\frac{\partial^{2} u}{\partial Y^{2}}+\frac{\partial^{2} u}{\partial T^{2}}\right)+u^{*} v=0 \\
& \mathrm{i} \frac{\partial v}{\partial Z}+\frac{1}{4}\left(\frac{\partial^{2} v}{\partial X^{2}}+\frac{\partial^{2} v}{\partial Y^{2}}+\sigma \frac{\partial^{2} v}{\partial T^{2}}\right)-\beta v+u^{2}=0 .
\end{aligned}
$$

Here, $T$, and $X, Y$ are the normalized temporal and transverse spatial coordinates, $Z$ is the normalized propagation distance, and $u, v$ are the $\mathbf{F H}$ and $\mathrm{SH}$ envelopes. A phase mismatch between the two harmonics is $\beta$, and $\sigma$ is the ratio of the GVD coefficients at the two frequencies. In the particular case $\sigma=1$, corresponding to identical FH and SH dispersions at the two frequencies, the model possesses an additional spatiotemporal spherical symmetry [35, 37].

We look for stationary solutions to Eqs. (1) in the form of $u=U(r, T)$ $\times \exp \left(\mathrm{i} \kappa_{1} Z+\mathrm{i} s \theta\right), v=V(r, T) \exp \left[2\left(\mathrm{i} \kappa_{1} Z+\mathrm{i} s \theta\right)\right]$, where $\theta$ is the polar angle in the transverse plane, $\kappa$ is the nonlinear wave number shift, and the integer $s$ is the vorticity number or "spin". The amplitudes $U$ and $V$ may be taken real, obeying the equations

$$
\begin{aligned}
& \frac{1}{2}\left(\frac{\partial^{2} U}{\partial r^{2}}+\frac{1}{r} \frac{\partial U}{\partial r}-\frac{s^{2}}{r^{2}} U+\frac{\partial^{2} U}{\partial T^{2}}\right)-\kappa_{1} U+U V=0 \\
& \frac{1}{4}\left(\frac{\partial^{2} V}{\partial r^{2}}+\frac{1}{r} \frac{\partial V}{\partial r}-\frac{4 s^{2}}{r^{2}} V+\sigma \frac{\partial^{2} V}{\partial T^{2}}\right)-\left(2 \kappa_{1}+\beta\right) V+U^{2}=0
\end{aligned}
$$


In Eqs. (2) $\sigma$ and $\beta$ are material and carrier-wave parameters, while $\kappa_{1}$ parametrizes the family of the stationary solutions. Note that solutions of this form assume that the phase helix is in the transverse spatial plane $(X, Y)$. One can also consider LB with a phase helix in a spatiotemporal plane. Because, in general the asymmetry parameter $\sigma \neq 1$, such solutions lack the axial symmetry; their analysis is much more complicated and therefore they are left beyond the scope of this work.

The total energy of LB is

$$
I=\iiint\left(|u|^{2}+|v|^{2}\right) \mathrm{d} X \mathrm{~d} Y \mathrm{~d} T \equiv I_{u}+I_{v}
$$

which is a conserved quantity. The other dynamical invariants are the Hamiltonian $H$, the momentum (equal to zero for the solution considered), and the angular momentum $L$ in the transverse plane. One readily finds from Eqs. (1), (2) that the Hamiltonian and the angular momentum of stationary spinning LB are related as follows:

$$
H=-\frac{1}{3} \kappa_{1} I+\frac{1}{3} \beta I_{v}
$$

and

$$
L=s I \text {. }
$$

In the particular case $s=1$ and $\sigma=0$ it is possible to get exact analytical solutions for spinning light bullets via a variational technique. To this aim, we choose the following Ansatz:

$$
\begin{aligned}
& U(r, T)=A r \exp \left(-a_{0} r^{2}-\alpha_{0} T^{2}\right), \\
& V(r, T)=B r^{2} \exp \left(-a_{0} r^{2}-2 \alpha_{0} T^{2}\right),
\end{aligned}
$$

where we have introduced the four free parameters $A, B, a_{0}$, and $\alpha_{0}$. The Lagrangean of the system to be minimized is $L=H+\kappa_{1} I$. Finally, we got the following result for the total energy and the Hamiltonian as a function of the wave number shift $\kappa_{1}$ and the phase mismatch $\beta$

$$
\begin{gathered}
I\left(\kappa_{1}, \beta\right)=\frac{\pi}{4 a_{0}^{2}} \sqrt{\frac{\pi}{4 \alpha_{0}}}\left(\sqrt{2} A^{2}+B^{2}\right), \\
H\left(\kappa_{1}, \beta\right)=\frac{\pi}{a_{0}^{3}} \sqrt{\frac{\pi}{4 \alpha_{0}}}\left(\frac{\sqrt{2}}{2} a_{0}^{2} A^{2}+\frac{\sqrt{2}}{8} a_{0} \alpha_{0} A^{2}+\frac{3}{16} a_{0} B^{2}+\frac{\beta}{8} B^{2}-\frac{2}{27} A^{2} B\right),
\end{gathered}
$$

where

$$
\begin{aligned}
& a_{0}=\frac{1}{9} \sqrt{\kappa_{2}}\left(\sqrt{\kappa_{2}+18 \kappa_{1}}-\sqrt{\kappa_{2}}\right), \\
& \alpha_{0}=\frac{2}{3}\left(\kappa_{1}+2 a_{0}\right), \\
& A^{2}=\frac{27}{16}\left(3 a_{0}+2 \kappa_{2}\right) B,
\end{aligned}
$$




$$
B=\frac{9 \sqrt{2}}{2} a_{0}\left(2 a_{0}+\kappa_{1}\right)
$$

and $\kappa_{2} \equiv 2 \kappa_{1}+\beta$.

We have numerically found one-parameter families of stationary $3 \mathrm{D}$ spinning-LB solutions, having the shape of a doughnut with a hole (phase dislocation) in the center, for different values of the GVD-asymmetry parameter $\sigma$. A standard band-matrix algorithm was used to deal with the corresponding two-point boundary-value problem. It was found that solutions exist provided that their energy exceeds a certain threshold. At the exact phase-matching point $(\beta=0)$, the threshold vanishes. For stationary solutions to decay exponentially at infinity, the wave number $\kappa_{1}$ has to obey the requirements $\kappa_{1}>0$ for $\beta \geq 0$, or $\kappa_{1}>-\beta / 2$ for $\beta<0$. We have found solitons only in the case when the $\mathrm{SH}$ dispersion is anomalous or zero, $\sigma \geq 0$.
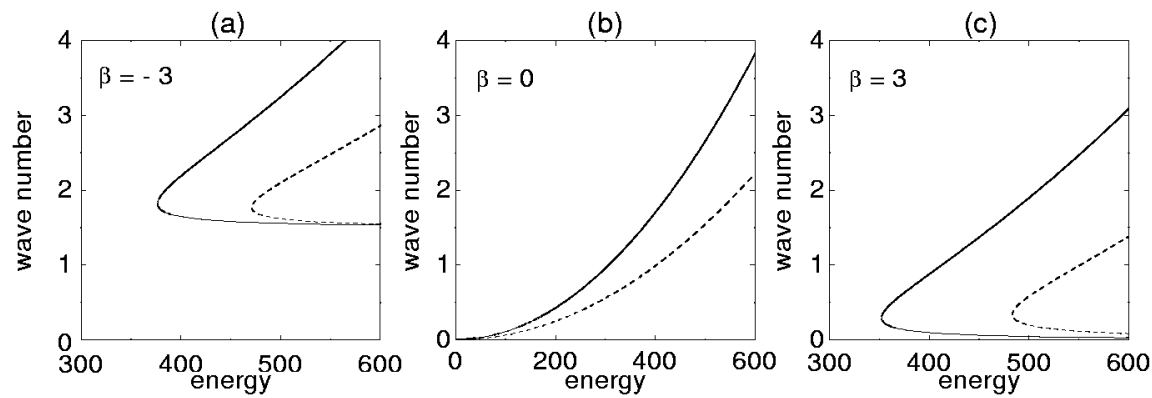

Fig. 1. The nonlinear wave number $\kappa_{1}$ vs. the energy $I$ for the light bullets with spin $s=1$ and $\sigma=0$ for the mismatch (a) $\beta=-3$, (b) $\beta=0$, and (c) $\beta=3$.
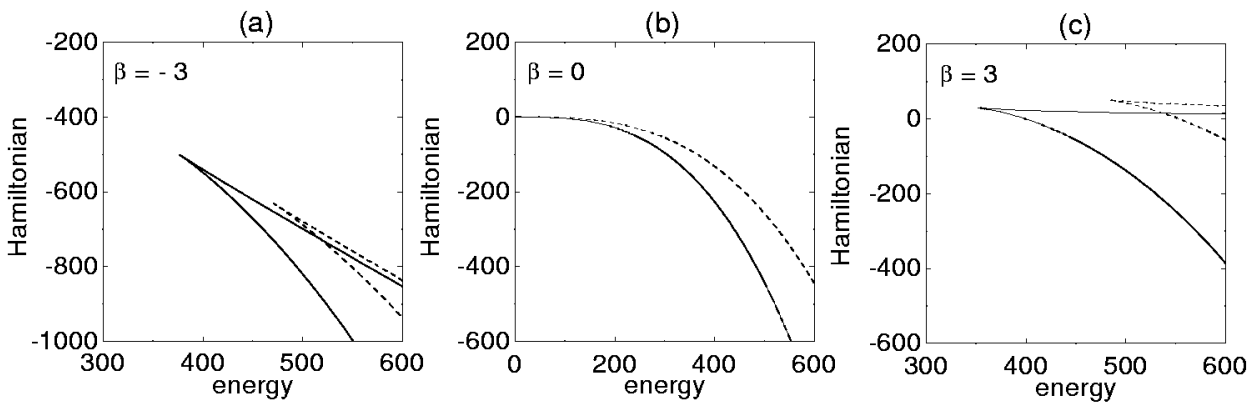

Fig. 2. The Hamiltonian $H$ vs. the energy $I$ for the light bullets with spin $s=1$ and $\sigma=0$ for the mismatch (a) $\beta=-3$, (b) $\beta=0$, and (c) $\beta=3$.

To characterize the LB solutions, in Figs. 1 and 2 we display the wave number $\kappa_{1}$ and the Hamiltonian $H$ of spinning LB with $s=1$ and $\sigma=0$ vs. its net energy $I$ for three representative values of the mismatch $\beta$. In Figs. 1 and 2 , the exact families of stationary LB are shown with full lines, whereas the approximate, variational solutions are shown with dashed lines. For larger values of the "spin" (e.g., $s=2$ ), the results are similar, although the threshold energies are higher. 
To conclude the discussion of the stationary solutions, we note that comparison with the simple variational approximation for the spinning LB, briefly described in Ref. [52], demonstrates that, although the variational approximation is not very accurate, it correctly describes the qualitative features of the shape of the spinning LB.

\section{Stability and possibility of observation of 3D light bullets}

Proceeding the stability simulations, we solved Eqs. (1), using the CrankNicolson scheme. The corresponding system of nonlinear equations was solved by means of the Picard iteration method, and the resulting linear system was treated by the Gauss-Seidel iterative scheme. For good convergence we needed, typically, five Picard iterations and eight Gauss-Seidel iterations. The transverse grid step sizes were $0.08 \leq \Delta X=\Delta Y=\Delta T \leq 0.12$ and, in most cases, the longitudinal step size was $\Delta Z=0.01$. To avoid distortion of the instability development under the action of the symmetry of the Cartesian computational mesh, we added initial perturbations that mimic random fluctuations in a real system (cf. Ref. [53]). Figure 3 displays the outcome of numerical simulations: the doughnut-like spinning LB are always unstable against azimuthal perturbations, which lead to breakup of the doughnuts into several nonspinning LB. In fact, this instability is quite similar to the theoretically [54] and experimentally [55] known instability of (2+1)D one-ring (fundamental) and two-ring (second-order) spatial bright vortex solitons in saturable and quadratically nonlinear media. It is also noteworthy that higher-order solitary waves in saturable media exhibit similar transverse instabilities that break their azimuthal symmetry $[53,56]$.

Three emerging fragments were found to have unequal energies in the $s=1$ case, whereas four fragments are found to have exactly equal energies in the $s=2$ case (see Fig. 3). After the breakup of the doughnut, the fragments fly out tangentially, rather than keeping to spiral (similar to what is known about the instability-induced breakup of the $(2+1) \mathrm{D}$ spatial vortex solitons [54]). Thus, the angular momentum of the doughnut "spinning" soliton is converted into the angular momenta of the emerging nonspinning fragments. Lastly, we have found that the number of the emerging fragments is roughly twice the original "spin" value $s$. The dependence of the number of the fragments on the other parameters is fairly weak.

The results of the direct dynamical simulations reported in this work must comply with the stability analysis based on Eqs. (1) linearized around the stationary spinning LB. In particular, similar to Refs. [54, 56], we expect that the number of the emerging fragments is determined by the "azimuthal number" of the perturbation mode having the largest growth rate. However, the corresponding eigenvalue problem turns out to be prohibitively complex, and is left beyond the scope of this work.

To create spinning solitons in the experiment, one can give the necessary vorticity to the original cylindrical laser pulse, passing it through a properly fabricated phase mask [55]. To estimate real physical parameters at which the spinning light bullets and their instability can be observed in the experiment (similar to the 

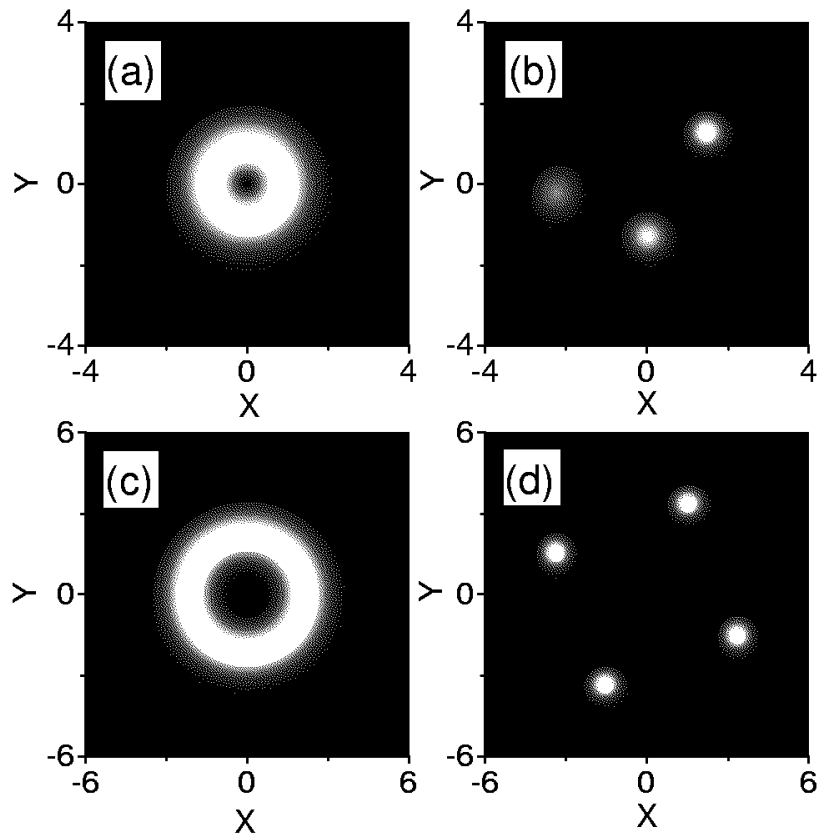

Fig. 3. Gray-scale contour plots illustrating the instability of the one-ring spinning light bullets. In (a) and (b) $s=1, \kappa_{1}=3$ while in (c) and (d) $s=2, \kappa_{1}=2.2$. The other parameters are $\sigma=2$ and $\beta=-3$. The propagation distance is $Z=8$ for $s=1$, and $Z=7$ for $s=2$. Only the fundamental-frequency component is shown.

experimental observation of the instability of the spatial vortex bright solitons in Ref. [55]), we can use the parameters at which the 2D LB in the SHG media were recently observed in Refs. [45]. In the $\mathrm{LiIO}_{3}$ optical crystal (in which the necessary temporal dispersion is induced artificially, by means of a grating), the light with the intensity $\sim 10 \mathrm{GW} / \mathrm{cm}^{2}$ self-traps in LB with characteristic temporal and spatial sizes $\sim 100 \mathrm{fs}$ and $40 \mu \mathrm{m}$, respectively. In the case of the 3D LB, the intensity should be, roughly, twice as large (see $[37,39]$ ). For the spinning LB with $s=1$, the energy is, typically, five times as large as for the zero-spin soliton with the same size. Thus, we arrive at an estimate for the energy of the spinning $3 \mathrm{D}$ LB of about $1 \mu \mathrm{J}$. These values of the physical parameters suggest that the experiment aimed to observe 3D LB should be quite feasible. For the physical interpretation of the results, it is also important to understand the real meaning of the propagation distances that appear in the above figures. A typical size of the $s=1 \mathrm{LB}$ is, in the dimensionless units, $\Delta x \sim 2$, hence the corresponding diffraction length is $z_{\mathrm{D}} \sim(\Delta x)^{2} \sim 4$. Thus, comparison with Fig. 3 and with other numerical results suggests that the full splitting of the spinning LB takes place after it has traveled a distance equal to a few diffraction lengths. On the other hand, a typical value of $z_{\mathrm{D}}$ in physical units is $\sim 3 \mathrm{~mm}$ [45]. This shows that the splitting process may be observed in available samples having lengths up to $3 \mathrm{~cm}[45]$. 


\section{Conclusions}

In conclusion, in the framework of the standard model of the type-I second-harmonic generation in a three-dimensional dispersive medium, we have found numerically one-parameter families of spatiotemporal doughnut-shaped spinning (vortex) solitons and we have made a comparison with the results obtained by using a simple variational method. All the spinning solitons show a strong symmetry-breaking azimuthal instability. The instability splits the spatiotemporal soliton into stable nonspinning light bullets, which fly out in tangential directions. The nonspinning three-dimensional spatiotemporal solitons are dynamically stable in most cases, hence in principle they may experimentally generated in quadratically nonlinear media.

\section{Acknowledgments}

Most of the research work briefly presented here have been carried out in collaboration with L.-C. Crasovan, F. Lederer, B.A. Malomed, D. Mazilu, I.V. Mel'nikov, N.-C. Panoiu, and L. Torner. I am deeply indebted to all of them. I also appreciate valuable discussions with $\mathrm{F}$.W. Wise on various issues considered in this work.

\section{References}

[1] G.I. Stegeman, D.J. Hagan, L. Torner, Opt. Quantum Electron. 28, 1691 (1996); Yu.S. Kivshar, in: Advanced Photonics with Second-Order Optically Nonlinear Processes, Eds. A.D. Boardman, L. Pavlov, S. Panev, Kluwer Academic, Dordrecht 1998, p. 451; L. Torner, in: Beam Shaping and Control with Nonlinear Optics, Eds. F. Kajzar, R. Reinisch, Plenum, New York 1998, p. 229; P.D. Drummond, K.V. Kheruntsyan, H. He, J. Opt. B, Quantum Semiclass. Opt. 1, 387 (1999); B. Malomed, in: Nonlinear Science at the Dawn of the 21st Century, Eds. P.L. Christiansen, M.P. Sorensen, A.C. Scott, Springer, Berlin 2000, p. 247.

[2] Yu.N. Karamzin, A.P. Sukhorukov, Sov. Phys. JETP 41, 414 (1976).

[3] A.A. Kanashov, A.M. Rubenchik, Physica D 4, 122 (1981).

[4] C.R. Menyuk, R. Schiek, L. Torner, J. Opt. Soc. Am. B 11, 2434 (1994).

[5] A.V. Buryak, Y.S. Kivshar, Opt. Lett. 19, 1612 (1994); Phys. Lett. A 197, 407 (1995).

[6] L. Torner, C.R. Menyuk, G.I. Stegeman, Opt. Lett. 19, 1615 (1994); L. Torner, Opt. Commun. 114, 136 (1995); L. Torner, D. Mihalache, D. Mazilu, N.N. Akhmediev, Opt. Lett. 20, 2183 (1995).

[7] A.V. Buryak, Y.S. Kivshar, V.V. Steblina, Phys. Rev. A 52, 1670 (1995).

[8] L. Torner, D. Mihalache, D. Mazilu, E.M. Wright, E.W. Torruellas, G.I. Stegeman, Opt. Commun. 121, 149 (1995).

[9] S.K. Turitsyn, JETP Lett. 61, 469 (1995).

[10] D. Mihalache, F. Lederer, D. Mazilu, L.-C. Crasovan, Opt. Eng. 35, 1616 (1996). 
[11] L. Bergé, V.K. Mezentsev, J.J. Rasmussen, J. Wyller, Phys. Rev. A 52, R28 (1995); L. Bergé, O. Bang, J.J. Rasmussen, V.K. Mezentsev, Phys. Rev. E 55, 3555 (1997).

[12] L. Torner, W.E. Torruellas, G.I. Stegeman, C.R. Menyuk, Opt. Lett. 20, 1952 (1995).

[13] A. De Rossi, S. Trillo, A.V. Buryak, Y.S. Kivshar, Opt. Lett. 22, 868 (1997).

[14] D.E. Pelinovsky, A.V. Buryak, Y.S. Kivshar, Phys. Rev. Lett. 75, 591 (1995); A.V. Buryak, Y.S. Kivshar, S. Trillo, Phys. Rev. Lett. 77, 5210 (1996).

[15] C. Etrich, U. Peschel, F. Lederer, B.A. Malomed, Y.S. Kivshar, Phys. Rev. E 54, 4321 (1996).

[16] L. Torner, D. Mazilu, D. Mihalache, Phys. Rev. Lett. 77, 2455 (1996); L. Torner, D. Mihalache, D. Mazilu, M.C. Santos, N.N. Akhmediev, J. Opt. Soc. Am. B 15, 1476 (1998).

[17] C. Etrich, U. Peschel, F. Lederer, B.A. Malomed, Phys. Rev. E 55, 6155 (1997); C. Etrich, U. Peschel, F. Lederer, D. Mihalache, D. Mazilu, Opt. Quantum Electron. 30, 881 (1998).

[18] D. Mihalache, D. Mazilu, L.-C. Crasovan, L. Torner, Opt. Commun. 137, 113 (1997); Phys. Rev. E 56, R6294 (1997).

[19] W.C.K. Mak, B.A. Malomed, P.L. Chu, Phys. Rev. E 55, 6134 (1997).

[20] P.D. Drummond, H. He, Phys. Rev. A 56, R1107 (1997); K.V. Kheruntsyan, P.D. Drummond, Phys. Rev. A 58, 2488 (1998).

[21] Y. Silberberg, Opt. Lett. 15, 1282 (1990).

[22] J.T. Manassah, P.L. Baldeck, R.R. Alfano, Opt. Lett. 13, 1090 (1988).

[23] A.B. Blagoeva, S.G. Dinev, A.A. Dreischuh, A. Naidenov, IEEE J. Quantum Electron. QE-27, 2060 (1991).

[24] M. Desaix, D. Anderson, M. Lisak, J. Opt. Soc. Am. B 8, 2082 (1991).

[25] R.H. Enns, D.E. Edmundson, S.S. Rangnekar, A.E. Kaplan, Opt. Quantum Electron. 24, S1295 (1992).

[26] D.E. Edmundson, R.H. Enns, Phys. Rev. A 51, 2491 (1995); Opt. Lett. 18, 1609 (1993)

[27] K. Hayata, M. Koshiba, Phys. Rev. Lett. 71, 3275 (1993); Phys. Rev. E 48, 2312 (1993).

[28] X.D. Cao, G.P. Agrawal, C.J. McKinstrie, Phys. Rev. A 49, 4085 (1994).

[29] K. Hayata, H. Higaki, M. Koshiba, Opt. Rev. 2, 233 (1995); IEICE T. Electron. E78C, 38 (1995).

[30] Y. Chen, J. Atai, Opt. Lett. 20, 133 (1995).

[31] R. McLeod, K. Wagner, S. Blair, Phys. Rev. A 52, 3254 (1995).

[32] H. He, M.J. Werner, P.D. Drummond, Phys. Rev. E 54, 896 (1996).

[33] V. Skarka, V.I. Berezhiani, R. Miklaszewski, Phys. Rev. E 56, 1080 (1997).

[34] A.W. Snyder, J.D. Mitchell, Opt. Lett. 22, 16 (1997).

[35] B.A. Malomed, P. Drummond, H. He, A. Berntson, D. Anderson, M. Lisak, Phys. Rev. E 56, 7425 (1997).

[36] S. Blair, K. Wagner, Opt. Quantum Electron. 30, 697 (1998).

[37] D.V. Skryabin, W.J. Firth, Opt. Commun. 148, 79 (1998). 
[38] D. Mihalache, D. Mazilu, B.A. Malomed, L. Torner, Opt. Commun. 152, 365 (1998).

[39] D. Mihalache, D. Mazilu, J. Dörring, L. Torner, Opt. Commun. 159, 129 (1999).

[40] M. Blaauboer, B.A. Malomed, G. Kurizki, Phys. Rev. Lett. 84, 1906 (2000); M. Blaauboer, G. Kurizki, B.A. Malomed, Phys. Rev. E 62, R57 (2000).

[41] I.V. Mel'nikov, D. Mihalache, N.-C. Panoiu, Opt. Commun. 181, 345 (2000).

[42] S. Raghavan, G.P. Agrawal, Opt. Commun. 180, 377 (2000).

[43] A. Desyatnikov, A.I. Maimistov, B.A. Malomed, Phys. Rev. E 61, 3107 (2000).

[44] D. Mihalache, D. Mazilu, L.-C. Crasovan, B.A. Malomed, F. Lederer, Phys. Rev. E 61, 7142 (2000).

[45] X. Liu, L.J. Qian, F.W. Wise, Phys. Rev. Lett. 82, 4631 (1999); X. Liu, K. Beckwitt, F. Wise, Phys. Rev. E 62, 1328 (2000).

[46] P. Di Trapani, D. Caironi, G. Valiulis, A. Dubietis, R. Danielius, A. Piskarskas, Phys. Rev. Lett. 81, 570 (1998); G. Valiulis, A. Dubietis, R. Danielius, D. Caironi, A. Visconti, P. Di Trapani, J. Opt. Soc. Am. B 16, 722 (1999).

[47] X. Liu, K. Beckwitt, F. Wise, Phys. Rev. E 61, R4722 (2000).

[48] D. Mihalache, D. Mazilu, B.A. Malomed, L. Torner, Opt. Commun. 169, 341 (1999).

[49] L. Torner, C.R. Menyuk, G.I. Stegeman, J. Opt. Soc. Am. B 12, 889 (1995); L. Torner, E.M. Wright, J. Opt. Soc. Am. B 13, 864 (1996).

[50] L. Torner, J.P. Torres, D. Artigas, D. Mihalache, D. Mazilu, Opt. Commun. 164, 151 (1999).

[51] D. Mihalache, D. Mazilu, L.-C. Crasovan, B.A. Malomed, F. Lederer, Phys. Rev. E 62, R1505 (2000).

[52] Y. Bakman, B. Malomed, in: Nonlinear Guided Waves and their Applications, OSA Technical Digest, Optical Society of America, Washington (DC) 1999, p. 389.

[53] D.E. Edmundson, Phys. Rev. E 55, 7636 (1997).

[54] L. Torner, D.V. Petrov, Electron. Lett. 33, 608 (1997); W.J. Firth, D.V. Skryabin, Phys. Rev. Lett. 79, 2450 (1997); D.V. Petrov, L. Torner, Opt. Quantum Electron. 29, 1037 (1997); D.V. Skryabin, W.J. Firth, Phys. Rev. E 58, 3916 (1998).

[55] D.V. Petrov, L. Torner, J. Martorell, R. Vilaseca, J.P. Torres, C. Cojocaru, Opt. Lett. 23, 1444 (1998).

[56] J.M. Soto-Crespo, D.R. Heatley, E.M. Wright, N.N. Akhmediev, Phys. Rev. A 44, 636 (1991); J. Atai, Y. Chen, J.M. Soto-Crespo, Phys. Rev. A 49, R3170 (1994). 